\title{
Methode zur automatisierten Topologiesynthese und Bewertung hybrider Antriebsstränge
}

Sebastian Ruoff ${ }^{1}$, Felix Kalt ${ }^{1}$, Katharina Bause ${ }^{1}$, Albert Albers ${ }^{1}$

${ }^{1}$ Institute of Product Engineering (IPEK),

Karlsruhe Institute of Technology (KIT), Germany

\section{Abstract}

The energy efficiency of hybrid electric vehicle drivetrains is in focus, as regulations force the vehicle manufacturers to reduce emissions. Therefore, in this paper, a method is described, that supports the developer in the early stage of product engineering [1], in the activities of detecting ideas by searching for alternative solutions. The method considers the system of objectives that has to be defined by the developer. Based on this, hybrid electric drivetrains topologies are synthesized automatically and investigated regarding their energy efficiency and performance. An automated rating process with an integrated weighting procedure that considers the system of objectives and a cost calculation, evaluates the topology investigations and gives the developer a topology ranking.

Keywords: topology synthesis, hybrid electric vehicle, early stage of product generation engineering, automated fuel consumption simulation 


\section{Motivation}

Durch die strengeren $\mathrm{CO}_{2}$-Vorgaben ist das Ziel der Fahrzeughersteller den Flottenverbrauch zu senken. Eine Möglichkeit zur Senkung ist die Elektrifizierung der Antriebsstränge. Auf Grund der hohen Kosten für rein elektrische Fahrzeuge, der langen Ladezeiten und der im Vergleich zu konventionell betriebenen Fahrzeugen geringen Gesamtreichweite, überwiegen die Vorteile hybridisierter Fahrzeugantriebsstränge. Diese sind zwar teurer als konventionelle Antriebsstränge, vereinen jedoch die Vorteile des lokal emissionsfreien Fahrens rein elektrischer Antriebsstränge mit den Vorteilen der hohen Reichweite und der schnellen Betankung konventioneller Fahrzeuge. Der Entwickler steht in der frühen Phase der Produktgenerationsentwicklung vor der Herausforderung mit wenig Information in einem riesigen Lösungsraum eine geeignete Antriebsstrangtopologie für das Fahrzeug im avisierten Nutzungsszenario zu identifizieren, die einen hohen Einfluss auf die Effizienz des Antriebsstranges hat [2].

\section{Forschungsproblem und Forschungsziel}

Mit steigender Anzahl an Komponenten im Antriebsstrang steigt die Anzahl möglicher Kombinationsmöglichkeiten und damit realisierbarer Topologien. Durch die verschiedenen Kombinationsmöglichkeiten der einzelnen Antriebsstrangkomponenten stellt sich die Frage, wie der Entwickler bei der Identifikation neuer Topologien unterstützt werden kann und wie diese beschrieben werden können. Die Anforderungen an den Entwickler, der große Lösungsmengen beherrschen muss, steigen und es stellt sich die Frage, wie die realisierbaren Topologien automatisiert untersucht und bewertet werden können.

\section{Verwendete Methoden, Vorgehensweise}

In der frühen Phase der Produktentstehung soll der Entwickler bei der Suche nach alternativen Lösungen, der Lösungsauswahl und der Tragweitenanalyse bei der Aktivität „Ideenfindung" nach dem integrierten Produktentstehungsmodell -iPeM [3] unterstützt werden. Im Rahmen der erarbeiteten Methode hat der Entwickler die Aufgabe das Zielsystem, wie in Kapitel 3.1 beschrieben, zu explizieren. Um neue Lösungen für den Antriebsstrang zu identifizieren schlagen die Autoren eine Methode vor, bei der Entwickler zu Beginn den Gestaltungsraum festlegt. Dazu muss der Entwickler die Anzahl der zulässigen Antriebsmaschinen und Getriebe in der neuen Topologie definieren. Je nach Definition können durch die mathematische Beschreibung von Antriebsstrangtopologien, unter der Verwendung der Graphentheorie, mehrere Tausend Antriebsstrangtopologien innerhalb einer Minute synthetisiert und auf mechanische Realisierbarkeit durch die Applikation von Regeln überprüft werden. 
Die Ergebnisse werden für jede Topologie in Form von Matrizen gespeichert, wie in Kapitel 3.2 beschrieben.

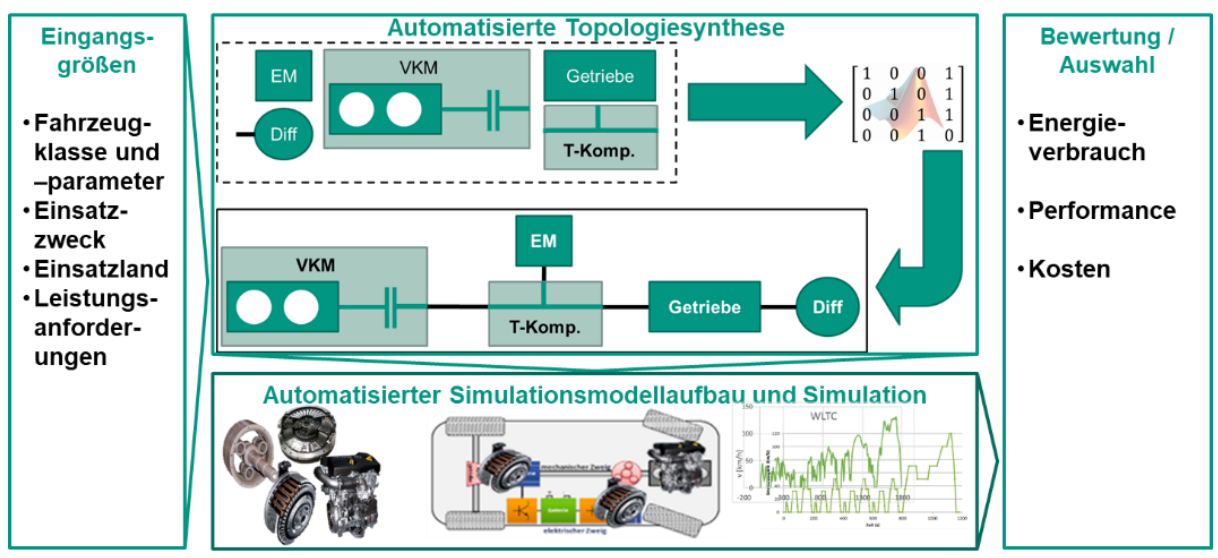

Bild 1: Visualisierung des Ablaufs der Methode [4]

Um den Entwickler bei der Lösungsauswahl zu unterstützen, werden die identifizierten Topologien anschließend hinsichtlich ihrer Eignung im definierten Zielsystem untersucht. Dazu werden Simulationsmodelle benötigt, die sowohl eine Aussage zu Leistungs- als auch zu Verbrauchswerten der einzelnen Topologien zulassen. Der Aufbau der Simulationsmodelle wird in Kapitel 3.3 expliziert. Da die Anzahl an synthetisierten Topologien sehr groß werden kann (bei bis zu drei elektrischen Maschinen, zwei Getrieben und einer Verbrennungskraftmaschine ca. 150 Kombinationsmöglichkeiten), werden die Simulationsmodelle automatisiert aufgebaut. Dazu werden die Matrizen mit den Topologieinformationen ausgelesen und für jede Topologie ein Simulationsmodell aufgebaut, welches auf einer Komponentenbibliothek basiert. Die verwendeten Antriebsmaschinen werden entsprechend der Leistungsvorgaben mit hinterlegten Kennfeldern parametrisiert und die Getriebeübersetzungen der Getriebe und die Daten des Restfahrzeugmodells, sowie des zu verwendenden Fahrzyklus automatisiert angepasst. Für jede Hybridklasse (parallel, seriell und leistungsverzweigt) ist in der Komponentenbibliothek eine allgemeingültige Betriebsstrategie hinterlegt. Mit dieser werden alle Topologien der jeweiligen Fahrzeugklasse simuliert, indem für jeden Zeitpunkt die bestmögliche Kombination der zur Verfügung stehenden Antriebsmaschinen und Gangkombinationen ausgewählt wird, um den Fahrerwunsch zu erfüllen. Dieser wird durch den Fahrzyklus vorgegeben. Nach der Simulation werden die simulierten Energieverbräuche und die erreichten Performancewerte in Kombination mit auf Kostenmodellen basierenden Antriebsstrangsystemkosten ausgewertet. Auf Basis 
des „Analytic Hierarchy" Prozesses werden mit anwendungsfallabhängigen Gewichtungsfaktoren Nutzwerte berechnet und auf deren Basis ein Ranking der Topologien erstellt [5]. Dieses Ranking unterstützt den Entwickler dabei, die besten vorgeschlagenen Topologien für seinen Anwendungsfall detaillierter zu untersuchen und die Tragweitenanalyse durchzuführen.

\subsection{Definition des Zielsystems}

Der Entwickler definiert das Zielsystem, indem er die Fahrzeugklasse und den Einsatzzweck des Fahrzeugs definiert, für das eine Antriebsstrangtopologie gefunden werden soll. Des Weiteren soll die Einsatzregion definiert werden, in der das Fahrzeug verkauft werden soll, um die gesetzlichen Rahmenbedingungen für das Einsatzgebiet zu identifizieren. Zudem soll definiert werden ob ein Benzin- oder Dieselmotor verwendet werden soll und ob es sich um einen autarken oder einen Plug-in Hybriden handelt. Neben diesen Informationen werden auch Zielgrößen für die Performance des Fahrzeuges benötigt, um den Leistungsbedarf abschätzen und damit die Antriebsmaschinen auswählen zu können. Basierend auf Vorgängerversionen werden Werte für die Berechnung der Fahrwiderstände, wie beispielsweise der $\mathrm{C}_{w}$-Wert oder die Stirnfläche des Fahrzeuges definiert.

\subsection{Automatisierte Topologiesynthese}

Um die Synthese hybrider Antriebsstränge automatisiert durchführen zu können, werden die Antriebsstrangtopologien mathematisch beschrieben. Unter Verwendung der Graphentheorie werden die Antriebsstränge durch Inzidenzmatrizen abgebildet. Grundlage für die Modellierung ist die Definition der Antriebsstrangkomponenten als „Knoten", im Sinne der Graphentheorie und die mechanischen Verbindungen zwischen den Antriebsstrangkomponenten werden als "Kanten" definiert [6]. Die Anzahl der mechanischen Anschlüsse einer Komponente definiert die Anzahl der Kanten, die in der mathemischen Beschreibung verbunden werden müssen. Antriebsmaschinen, wie die Verbrennungskraftmaschine und der Elektromotor sind dadurch gekennzeichnet, dass sie einen mechanischen Anschluss haben und daher mit einer Kante modelliert werden. Ein Getriebe hat einen mechanischen Eingang und einen Ausgang und wird daher als Knoten mit zwei Kanten modelliert. Um die Energieeffizienz und die Performance der jeweiligen Topologie im gewählten Nutzungsfall zu bewerten, wird ein Einspurmodell implementiert, welches die Längsdynamik des Fahrzeuges abbildet. Das Restfahrzeugmodell beinhaltet neben dem Differential die Seitenwellen und die Räder des Antriebsstranges, weshalb das Differential lediglich mit einem mechanischen Anschluss zum Antriebsstrang modelliert wird. 
In Bild 2 ist die schematische Darstellung eines hybriden Antriebsstranges dargestellt. Es ist zu erkennen, dass die koaxiale Anbindung einer elektrischen Maschine zwischen Verbrennungsmotor und Getriebe durch einen mechanischen Anschluss an der elektrischen Maschine nicht realisierbar ist.

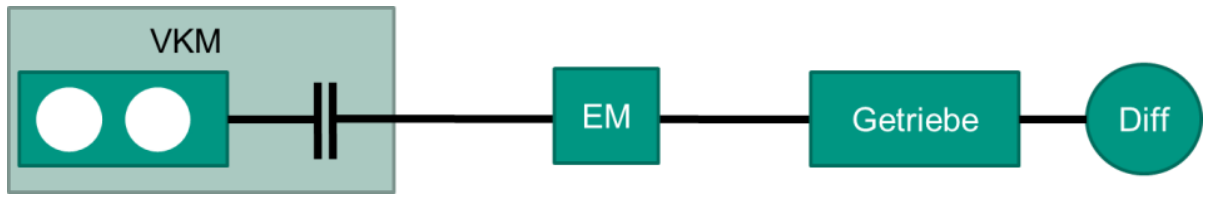

Bild 2: Schematische Darstellung eines P2-Hybrid-Anrtiebsstranges

Eine Möglichkeit wäre die Einführung zweier Modellierungen für eine elektrische Maschine, eine mit einem mechanischen Anschluss und eine mit zwei mechanischen Anschlüssen. Um Fehler in der Matrizengenerierung zu vermeiden, wird eine mechanische Schnittstelle eingeführt, die den Anschluss dreier Komponenten erlaubt. Diese wird im Folgenden als "T-Komponente" bezeichnet. Damit genügt die Modellierung der elektrischen Maschine mit einer mechanischen Schnittstelle und es ist möglich den korrekten Einbau der elektrischen Maschine mittels eindeutiger Regeln zu überprüfen. In Bild 3 ist ein hybrider Antriebsstrang mit eingebauter T-Komponente dargestellt.

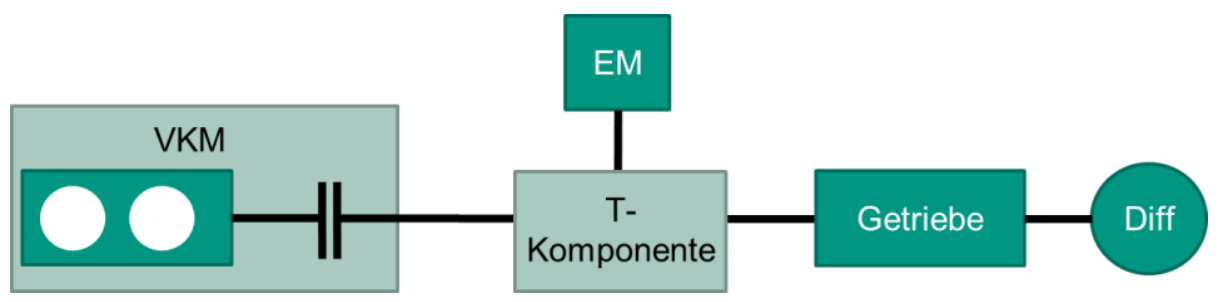

Bild 3: Schematische Darstellung eines P2-Hybrid-Antriebsstranges mit TKomponente

Eine T-Komponente kann für mehrere mechanische Ausprägungen im Antriebsstrang stehen: Zum einen kann eine verlustfreie Drehmomentenaddition über eine koaxiale Anbindung der elektrischen Maschine realisiert werden. Es sind zudem weitere mechanische Ausprägungen zur Drehmomentenaddition, wie beispielsweise eine Getriebestufe mit der Übersetzung 1, ein Kettentrieb oder ähnliches möglich. Im Rahmen der Simulation wird jedoch eine verlustfreie koaxiale Anbindung der elektrischen Maschine bei Drehmomentenaddition angenommen. Die Übertragung der Funktion in die Gestalt des Antriebsstranges erfolgt erst im Nachgang der Methode und ist für die Simulation nicht relevant. 
Zum anderen kann eine Drehzahladdition über ein Planetengetriebe erfolgen. Dabei sind sechs verschiedene Verschaltungen der angrenzenden Komponenten am Planetengetriebe realisierbar, da es sechs Permutationsmöglichkeiten an den drei Anschlüssen Sonnenrad, Steg und Hohlrad gibt. Die Drehzahladdition kann auf Grund der Verluste im Planetengetriebe nicht verlustfrei angenommen werden.

Die in Bild 3 dargestellte Topologie kann durch eine Inzidenzmatrix, wie in Tabelle 1 dargestellt, beschrieben werden. Dabei werden in jeder Zeile die mechanischen Verbindungen zwischen den verschiedenen Komponenten beschrieben. Jede Zeile steht für eine mechanische Verbindung und hat daher einen Anfang und ein Ende und somit genau zwei Einträge. Die letzte Zeile definiert die letzte Komponente im Leistungsfluss. In dieser Zeile wird der Anschluss an das Differenzial beschrieben, weshalb dort nur ein Eintrag in der Spalte der Komponente, die an das Differenzial angeschlossen ist, vorgenommen wird. Eine weitere Regel lautet, dass die Summe der Einträge in einer Spalte gleich der Anzahl der mechanischen Anschlüsse der jeweiligen Komponente sein muss.

Tabelle 1: Mathematische Beschreibung der P2-Hybridtopologie aus Bild 3

\begin{tabular}{|l|l|l|l|l|}
\hline Inhalt & VKM & EM & Getriebe & T-Komponente \\
\hline Kante 1 & 1 & 0 & 0 & 1 \\
\hline Kante 2 & 0 & 1 & 0 & 1 \\
\hline Kante 3 & 0 & 0 & 1 & 1 \\
\hline Kante 4 & 0 & 0 & 1 & 0 \\
\hline
\end{tabular}

Durch die Anwendung der Graphentheorie, zur mathematischen Beschreibung von Antriebsstrangtopologien, können Constraint Satisfaction Probleme [7] durch Regeln beschrieben und durch Algorithmen gelöst werden. Damit können die hybriden Antriebsstrangtopologien automatisiert für definierte Lösungsraume generiert und anschließend in Matrizen gespeichert werden.

\subsection{Systemanalyse durch die virtuelle Simulation}

Die zuvor erstellten Matrizen, mit denen der mechanische Aufbau der Antriebsstrangtopologien beschrieben wird, werden im nächsten Schritt ausgelesen und die Information verwendet, um automatisiert Simulationsmodelle für 
jede Topologie zu generieren. Mit diesen Simulationsmodellen können Untersuchungen zum Kraftstoffverbrauch und zur Performance des Antriebsstranges durchgeführt werden.

Um die Untersuchungen in Bezug auf das definierte Zielsystem durchführen zu können, müssen zunächst die erforderlichen Leistungen der Verbrennungskraftmaschine und der elektrischen Maschine( $n$ ) ermittelt werden. Dazu werden die Leistungsanforderungen an den Antriebsstrang auf Basis der Höchstgeschwindigkeits- und Beschleunigungsvorgaben unter Berücksichtigung der Fahrwiderstände aus dem Zielsystem herangezogen. Basierend auf den Leistungsanforderungen werden die Kennfelder der Antriebsmaschinen aus einer Bibliothek ausgewählt und die Simulationsmodelle parametriert. Des Weiteren werden die Getriebeübersetzungen für die verschiedenen Getriebe berechnet und im Simulationsmodell definiert. Die Kapazität der Batterie wird auf Basis der Anforderungen an die elektrische Reichweite ermittelt und das Batteriemodell ebenfalls entsprechend parametriert. Das Restfahrzeugmodell wird mit den Daten aus dem Zielsystem parametriert und die Betriebsstrategie in Abhängigkeit der Topologieklasse (parallel, seriell oder leistungsverzweigt) ausgewählt. Die Betriebsstrategie wählt zu jedem Zeitpunkt den energieoptimalen Fahrmodus in Abhängigkeit des SoC und der aktuellen Leistungsanforderung aus. Vor der Simulation wird in einem Preprocessing ermittelt bei welcher Fahrgeschwindigkeit und welcher Beschleunigungsanforderung, welche Kombination aus Gangwahl und Drehmomentkombination der verschiedenen Antriebsmaschinen aus energischer Sicht am besten geeignet ist. Entsprechend wird zu jedem Zeitpunkt überprüft welcher Fahrzustand vorliegt, welche Anforderungen aus dem Fahrzyklus und dem Fahrermodell an das Fahrzeug gestellt werden und die Maschinen und das Getriebe entsprechend angesteuert, sofern es der Ladezustand der Batterie zulässt. Ist der Ladezustand zu gering, wird rein konventionell gefahren.

Im Rahmen der Verbrauchssimulation wird in Abhängigkeit des Zielsystems der entsprechende Fahrzyklus hinterlegt und dieser mit voller und leerer Batterie durchfahren, um anschließend den normgerechten Kraftstoffverbrauch berechnen zu können. Zudem werden Beschleunigungs- und Höchstgeschwindigkeitssimulationen durchgeführt, mit Hilfe derer Ergebnisse die Erfüllung der Anforderungen in der automatisierten Bewertung hinsichtlich Performance überprüft werden kann.

\subsection{Automatisierte Bewertung}

Um dem Entwickler die Identifikation der am besten geeigneten Topologie für das definierte Zielsystem zu ermöglichen, werden Kriterien benötigt, die für 
die Bewertung der Topologien herangezogen werden. Die Hauptkriterien „Energieverbrauch" und "Performance" setzen sich aus verschiedenen Unterkriterien zusammen. Die "Kosten" für die jeweilige werden auf Basis von Kostenmodellen für die Antriebsmaschinen, Getriebe und die Batterie ermittelt. Mit dem „AnaIytic Hierarchy Process" [8] werden die Unterkriterien zu einer Gesamtbewertung mit einer Gewichtung verrechnet. Da unterschiedliche Zielsysteme unterschiedliche Anforderungen an den Antriebsstrang enthalten, muss die Gewichtung der Kriterien zur Identifikation der Gesamtbewertung an das jeweilige Zielsystem angepasst werden. Bei einem Stadtfahrzeug spielt die Performance beispielsweise eine geringere Rolle als bei einem Sportfahrzeug. Des halb werden in der Methode unterschiedliche Gewichtungen hinterlegt, die automatisiert, je nach Zielsystem, verwendet werden können, um das Ergebnis auf den jeweiligen Anwendungsfall abstimmen zu können. Das Gesamtergebnis wird dem Entwickler als Nutzwert auf einer Skala zwischen 0 und 100 ausgegeben. [5]

\section{Verifizierung der Simulationsergebnisse}

Zur Verifizierung der Simulationsergebnisse werden verschiedene Referenzfahrzeuge gewählt und diese über die Topologiesynthese und Simulation nachgebildet. Die Verifizierung erfolgt über die Gegenüberstellung und Interpretation der Bewertungsgrößen. Exemplarisch ist in Tabelle 2 der Vergleich des BMW 330e aus dem Jahr 2019 mit der Simulation eines Mittelklassefahrzeuges mit dem Einsatzzweck als „Alltagsfahrzeug" mit bevorzugtem Streckenprofil "Autobahn" gegenübergestellt. Die Fahrzeugparameter der Simulation wurden denen des Vergleichsfahrzeuges angepasst und es wurden Antriebsmaschinen der gleichen Leistungsklasse eingesetzt.

Es ist eindeutig zu erkennen, dass die Abweichung beim Verbrauch im NEFZ bei ca. $5 \%$ liegen, wohingegen die Abweichung beim Verbrauch im WLTP mit ca. $20 \%$ deutlich höher ausfällt. Demgegenüber steht allerdings auch ein erhöhter elektrischer Energieverbrauch in Höhe von ca. 15\%, weshalb davon ausgegangen werden kann, dass das Fahrzeug in der Simulation des WLTP häufiger elektrisch gefahren ist und deshalb einen erhöhten elektrischen Verbrauch und einen geringeren Kraftstoffverbrauch aufweist. Insgesamt kann jedoch aus dem Vergleich die Schlussfolgerung gezogen werden, dass der gesamte Energieverbrauch in der Simulation in einer realistischen Größenordnung liegt. Insbesondere unter Berücksichtigung der Tatsache, dass auf Grund des frühen Entwicklungsstadiums, in dem die Methode eingesetzt werden soll, die eingesetzten Antriebsmaschinen lediglich im gleichen Leistungsbereich liegen und nicht bekannt ist wie das genaue Kennfeld der Antriebsmaschinen des Vergleichsfahrzeuges aussieht. 
Tabelle 2: Gegenüberstellung der Simulationsergebnisse mit Fahrzeugdaten eines Vergleichsfahrzeuges (BMW 330e Modelljahr 2019)

\begin{tabular}{|l|l|l|}
\hline Vergleichsgröße & Referenzfahrzeug & $\begin{array}{l}\text { Gleiches Hybridfahr- } \\
\text { zeug aus Topologie- } \\
\text { synthese }\end{array}$ \\
\hline Verbrauch NEFZ & $1,6 \mathrm{l} / 100 \mathrm{~km}$ & $1,68 \mathrm{l} / 100 \mathrm{~km}$ \\
\hline Verbrauch WLTP & $1,4 \mathrm{l} / 100 \mathrm{~km}$ & $1,14 \mathrm{l} / 100 \mathrm{~km}$ \\
\hline Verbrauch WLTP elektr. & $15,9 \mathrm{kWh}$ & $17,7 \mathrm{kWh}$ \\
\hline Leistung VKM & $215 \mathrm{~kW}$ & $214 \mathrm{~kW}$ \\
\hline Leistung EM & $83 \mathrm{~kW}$ & $86 \mathrm{~kW}$ \\
\hline Gewicht & $1815 \mathrm{~kg}$ & $1858 \mathrm{~kg}$ \\
\hline
\end{tabular}

\section{Ergebnisse und Diskussion}

Mit Hilfe der vorgestellten Methode erhalten Entwickler innerhalb eines Tages Ergebnisse für mehr als 100 verschiedene Topologien, die bereits automatisiert bewertet wurden und nach Nutzwert sortiert aufgelistet sind. Des Weiteren stehen zu jeder Topologie detaillierte Informationen zu Kraftstoffverbrauch, Performance und Kosten zur Verfügung, die der Entwickler analysieren kann, um die für ihn beste Topologie auszuwählen. Zudem gibt es unter den untersuchten Topologien mechanisch ähnliche Topologien, die in Kombination effizienter sein könnten. Für diese Topologien wurde noch keine Möglichkeit implementiert, automatisiert weitere Untersuchungen durchzuführen. Im Rahmen einer Studie wurde die Anwendbarkeit der Methode untersucht und es konnte gezeigt werden, dass das unterstützende Softwaretool für die Suche nach geeigneten Antriebsstrangtopologien geeignet ist und die Teilnehmer der Studie die Betrachtung ähnlicher Topologien zur Steigerung der Gesamteffizienz für sinnvoll erachten [9]. Im weiteren Verlauf des Produktentstehungsprozesses erfolgt die Aktivität der Modellierung von Prinzip und Gestalt. Dazu kann der Entwickler auf den vorgeschlagenen Topologien aufbauen und für eine oder mehrere Topologien weitere Untersuchungen und Entwicklungen vornehmen. 


\section{Literatur}

[1] Albers, A. et al.: "Die Frühe Phase der PGE - Produktgenerationsentwicklung", Stuttgarter Symposium für Produktentwicklung, 2017.

[2] Li, X; Williamson, S.: "Comparative Investigation of Series and Parallel Hybrid Electric Vehicle (HEV) Efficiencies Based on Comprehensive Parametric Analysis", IEEE, Vehicle Power and Propulsion conference, 2007.

[3] Albers, A. et al.: "iPeM - Integrated Product Engineering Model in Context of Product Generation Engineering", Procedia CIRP, Vol 50, 2016, 100-105.

[4] Ruoff, S.: "Topology synthesis of hybrid electric vehicle drivetrains in the context of the integrated Product engineering Model", Procedia EVS 30 Symposium, Stuttgart, 2017.

[5] Ruoff, S.; Busch, M.; Bause, K.: "Evaluation of new hybrid electric vehicle drivetrain topologies", Procedia EVS31 Symposium, Kobe, 2018.

[6] Krischke, A., Röpcke, H.: "Graphen und Netzwerktheorie: Grundlagen Methoden - Anwendungen; mit zahlreichen Beispielen", Fachbuchverlag Leipzig im Hanser Carl-Hanser-Verlag, München, 2015.

[7] Dechter, R.; Cohen, D.: "Constraint processing", Morgan Kaufmann Publishers, San Francisco, 2003.

[8] Zimmermann, H-J.; Gutsche, L.: "Multi-Criteria Analyse. Einführung in die Theorie der Entscheidungen bei Mehrfachzielsetzungen", Springer Verlag, Berlin, Heidelberg, 1991.

[9] Ruoff, S.; Schwarz, A.-M.; Bause, K.: "Investigation of the necessity of methodical support of the developer to combine the advantages of two hybrid electric topologies in order to increase the number of realizable functions", Procedia EVS 32 Symposium, Lyon, 2019. 